\title{
Nonlinear Optimal Control of Large-Scale Systems; Part II - Interaction Balance Principle
}

\author{
N. SADATI and E. DEHGHAN MARVAST \\ Intelligent Systems Laboratory, \\ Department of Electrical Engineering, \\ Sharif University of Technology, \\ Tehran, Iran. \\ e-mail: sadati@sina.sharif.edu
}

\begin{abstract}
In this part, similar to Part I of this paper, a new two-level method for nonlinear optimal control of large scale systems is introduced. This approach is based on Interaction Balance Principle for coordination of large-scale systems. In the first level, the optimization problems are solved for nonlinear dynamics using a gradient method, and in the second level, the coordination is done using the gradient of errors to improve the convergence rate in compare to the classical Goal Coordination method and obtain the overall optimal solution.

The efficacy and advantages of the new approach is shown in an application example.
\end{abstract}

\section{INTRODUCTION}

In control of large-scale systems, two-level methods are very attractive, because the control problem can be solved in several subsystems and this leads to better distribution of memory storage and faster computation. In the optimal control problem for large-scale systems, the problem is to minimize a cost function subject to the systems dynamics.

For using a two-level method the system should be divided into several interconnected subsystems and the cost function should be divided into several cost functions for each subsystem. Minimization of these cost functions for each subsystem leads to several sub-problems. For coordination of these sub-problems two coordination principles have been proposed by Mesarovic et al. [1],[2] named Interaction Balance Principle and Interaction Prediction Principle. In using these principles for control of large-scale systems, these systems are decomposed into several subsystems with interaction inputs from each other. So, each subsystem solve its own optimization problem and a high level controller coordinate these low level optimizers to solve the overall problem.

In applying the Interaction Balance Principle, the high level coordinator modifies the infimal performance function for each subsystem; compare the optimal interaction inputs computed by low-level subsystems by real interactions then provide new modified performance function to decrease the coordination error to zero.

Classical Goal Coordination method, based on Interaction Balance Principle has been applied to linear and nonlinear problems [3]-[5].

In this paper the Goal Coordination method is used for coordination and a new coordination algorithm based on the gradient of interaction errors is used to improve the convergence rate of the solution [6]-[8].
In the sequel, a brief description of Goal Coordination and Interaction Balance Principle is given in section II. Then, decomposition of the overall problem and the firstlevel optimizations are described in sections III and IV, respectively. In section $\mathrm{V}$, a new gradient-based coordinator with high-speed convergence is postulated and finally, the simulation results are shown followed by concluding remarks in sections VI and VII, respectively.

\section{GOAL COORDINATION AND INTERACTION BALANCE PRINCIPLE}

As mentioned above, the two principles for coordination of large-scale systems are Interaction Balance and Interaction Prediction Principles. Since the Goal Coordination based on Interaction Balance Principle is used in this part of the paper, this principle is introduced in continue.

Let $B$ be a set such that each $\beta$ in $B$ specifies, for each $\mathrm{i}=0,1, . ., N$, a performance function $G_{i \beta}: U_{i} \times Z_{i} \times Y_{i} \rightarrow V$ which is a modification of the original $G_{\mathrm{i}}$. Let the mapping $g_{i \beta}$ be defined on $U_{i} \times Z_{\mathrm{i}}$ in terms of $P_{\mathrm{i}}$ and $G_{i \beta}$, as in (1)

$$
\begin{aligned}
& g_{i \beta}: U_{i} \times Z_{i} \rightarrow V \\
& g_{i \beta}\left(u_{i}, z_{i}\right)=G_{i \beta}\left(u_{i}, z_{i}, P\left(u_{i}, z_{i}\right)\right)
\end{aligned}
$$

For each $\beta$ in $B$, the infimal control problem is to find the pair $\left(\hat{u}_{i}, \hat{z}_{i}\right)$ in $U_{i} \times Z_{\mathrm{i}}$ such that

$$
g_{i \beta}\left(\hat{u}_{i}, \hat{z}_{i}\right)=\min _{U_{i} \times Z_{i}} g_{i}\left(u_{i}, z_{i}\right)
$$

Minimization is over both sets $U_{i}$ and $Z_{\mathrm{i}}$ and the interaction inputs are treated as free variables.

Let $\beta$ in $B$ be given; let $\hat{z}_{1}(\beta), \cdots, \hat{z}_{N}(\beta)$ be the interaction inputs required by the infimal controllers to achieve the local optimum, let $z_{1}(\beta), \cdots, z_{N}(\beta)$ be the interaction inputs that occur if the controls $\hat{u}_{1}(\beta) \cdots \hat{u}_{2}(\beta)$ are implemented. The overall optimum is achieved if the actual interaction inputs are precisely those obtained by the local optimization for each subsystem; $i=1, \ldots, N[1]$, so that

$$
\widehat{z}_{i}(\beta)=z_{i}(\beta)
$$

If the Interaction Balance Principle applies, the supremal control action is to find $\beta$ in $B$ such that $\varepsilon_{i}=\widehat{z}_{i}(\beta)-z_{i}(\beta)=0$ 
for all subsystems.

Fig. 1 shows the application of Interaction Balance Principle for coordination of two sub-problems.

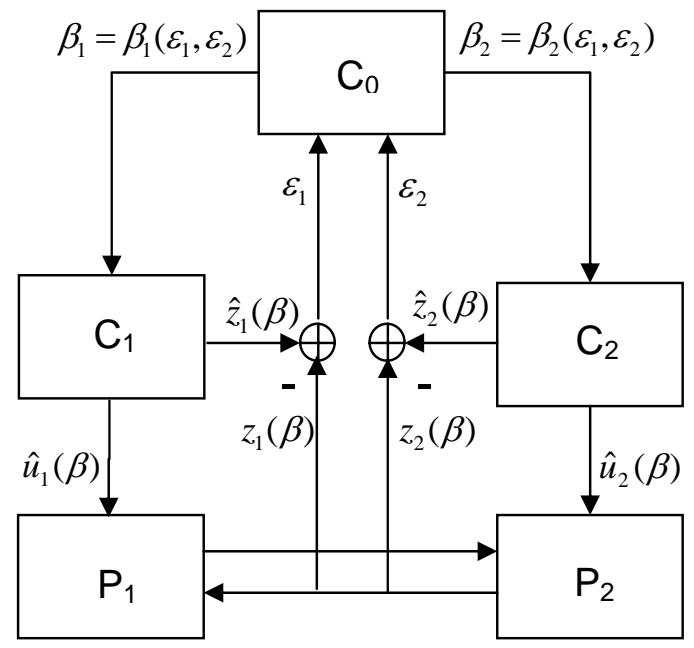

Fig. 1 Application of Interaction Balance Principle for coordination of two sub-problems

Now, the remainder is to decompose the overall optimization problem into several sub-problems and coordinate sub-problems to gain the overall answer.

Suppose we have a general nonlinear system described by the following state space equation.

$$
\underline{x}(k+1)=\underline{f}(\underline{x}(k), \underline{u}(k))
$$

where $\underline{X}$ is the state vector, $\underline{u}$ is the control input vector and $f$ is a continuously double differentiable analytical vector function.

The problem is to find $\underline{u}$ which minimize the cost function given by

$$
J=G_{n+1}(\underline{x}(n+1))+\sum_{k=0}^{n} G_{k}(\underline{x}(k), \underline{u}(k))
$$

In this equation, $G_{k}$ is a general nonlinear scalar function of its arguments.

\section{DECOMPOSITION OF THE OVERALL PROBLEM}

Let assume that the overall system is a combination of $N$ interconnected subsystems and each subsystem has a state space equation as below:

$$
\begin{aligned}
& \underline{x}_{i}(k+1)=\underline{f}_{i}\left(\underline{x}_{i}(k), \underline{u}_{i}(k), \underline{z}_{i}(k)\right) \\
& \underline{x}_{i}(0)=\underline{x}_{i 0}
\end{aligned}
$$

where $\underline{X}_{i}$ is the state vector, $\underline{u}_{i}$ the input and $\underline{Z}_{i}$ is the interaction input of the $i$ th subsystem that is assumed to be a nonlinear function of the other subsystems state vector.

$$
\underline{z}_{i}(k)=H_{i}(\underline{x}(k))
$$

In Goal Coordination method, it is necessary for function
$H_{i}$ to be separable, i.e.

$$
\underline{Z}_{i}(k)=H_{i}(\underline{x}(k))=\sum_{j=1}^{N} H_{i j}\left(\underline{x}_{j}(k)\right)
$$

The interaction relations, which can be shown as $\underline{z}(k)=H(\underline{x}(k))$ are considered to be constraints for optimization problem (5), so we can define the Lagrangian as

$$
\begin{aligned}
L & =G_{n+1}(\underline{x}(n+1))+\sum_{k=0}^{n} G_{k}(\underline{x}(k), \underline{u}(k)) \\
& +\sum_{k=0}^{n+1} \underline{\beta}^{T}(k)(\underline{z}(k)-H(\underline{x}(k)))
\end{aligned}
$$

In this equation, $\beta \mathrm{s}$ are the Lagrange multipliers which are used as coordination parameters in Goal Coordination method.

Lagrangian (8) can be change into $N$ separated Lagrangian for each subsystem. i.e.

$$
\begin{aligned}
L_{i} & =G_{i_{n+1}}\left(\underline{x}_{i}(n+1), \underline{Z}_{i}(n+1)\right)+\sum_{k=0}^{n} G_{i_{k}}\left(\underline{x}_{i}(k), \underline{u}_{i}(k), \underline{Z}_{i}(k)\right) \\
& +\sum_{k=0}^{n+1}\left(\underline{\beta}_{i}^{T}(k) \underline{Z}_{i}(k)-\sum_{j=1}^{N} \underline{\beta}_{j}^{T}(k) H_{j i}\left(\underline{x}_{i}(k)\right)\right)
\end{aligned}
$$

In each subsystem, the problem is to solve the following problem with known $\beta$ coming from the high level coordinator

$$
\begin{aligned}
& \min _{x_{i}, u_{i}, z_{i}} L_{i}=G_{i_{n+1}}\left(\underline{x}_{i}(n+1), \underline{Z}_{i}(n+1)\right)+\sum_{k=0}^{n} G_{i_{k}}\left(\underline{x}_{i}(k), \underline{u}_{i}(k), \underline{Z}_{i}(k)\right) \\
& +\sum_{k=0}^{n+1}\left(\underline{\beta}_{i}^{T}(k) \underline{z}_{i}(k)-\sum_{j=1}^{N} \underline{\beta}_{j}^{T}(k) H_{j i}\left(\underline{x}_{i}(k)\right)\right) \\
& \text { s.t. } \quad \underline{x}_{i}(k+1)=\underline{f}_{i}\left(\underline{x}_{i}(k), \underline{u}_{i}(k), \underline{z}_{i}(k)\right)
\end{aligned}
$$

and in the second level the problem is to update the coordination parameters, $\beta$, such that the interaction errors; $\underline{z}_{i}(k)-H_{i}(\underline{x}(k))$, become zero [1].

\section{OPTIMIZATION OF THE FIRST LEVEL}

In the first level the problem is to solve the optimization problem described in (10), by assuming constant known values of $\underline{\beta}$ from the second level. So by adding the following term

$$
\underline{\lambda}_{i}^{T}(k)\left[\underline{x}_{i}(k+1)-\underline{f}_{i}\left(\underline{x}_{i}(k), \underline{u}_{i}(k), \underline{\underline{z}}_{i}(k)\right)\right\rfloor
$$

to the Lagrangian, the new Lagrangian can be written as

$$
\begin{aligned}
L_{i}= & G_{i_{i_{n+1}}}\left(\underline{X}_{i}(n+1), \underline{z}_{i}(n+1)\right)+\sum_{k=0}^{n} G_{i_{k}}\left(\underline{x}_{i}(k), \underline{u}_{i}(k), \underline{z}_{i}(k)\right) \\
& +\sum_{k=0}^{n+1}\left(\underline{\beta}_{i}^{T}(k) \underline{z}_{i}(k)-\sum_{j=1}^{N} \underline{\beta}_{j}^{T}(k) H_{j i}\left(\underline{x}_{i}(k)\right)\right) \\
& +\sum_{k=0}^{n} \underline{\lambda}_{i}^{T}(k)\left[\underline{X}_{i}(k+1)-\underline{f}_{i}\left(\underline{x}_{i}(k), \underline{u}_{i}(k), \underline{z}_{i}(k)\right)\right]
\end{aligned}
$$


To solve this problem, an algorithm based on optimality necessary conditions can be used as below [10]:

1. Choose initial values for $\underline{u}_{i}(0)$ to $\underline{u}_{i}(n)$, and $\underline{Z}_{i}(0)$ to $\underline{Z}_{i}(n+1)$.

2. Use known $\underline{x}_{i 0}$ and values for $\underline{u}_{i}(k)$ and $\underline{z}_{i}(k)$ to compute the values of $\underline{x}_{i}(1)$ to $\underline{x}_{i}(n+1)$, using system's state space equations.

3. Calculate $\underline{\lambda}_{i}(k)$ for $k=n, n-1, \ldots, 0$ backward in time, by using the following necessary conditions:

$$
\begin{aligned}
\underline{\lambda}_{i}(n) & =-\frac{\partial G_{i_{n+1}}\left(\underline{x}_{i}(n+1), \underline{z}_{i}(n+1)\right)}{\partial \underline{x}_{i}(n+1)} \\
& +\sum_{i=1}^{N}\left(\frac{\partial H_{j i}(n+1)}{\partial \underline{x}_{i}(n+1)}\right)^{T} \underline{\beta}_{j}(n+1) \\
\underline{\lambda}_{i}(k-1) & =-\frac{\partial G_{i_{k}}\left(\underline{x}_{i}(k), \underline{u}_{i}(k), \underline{z}_{i}(k)\right)}{\partial \underline{x}_{i}(k)} \\
& +\sum_{i=1}^{N}\left(\frac{\partial H_{j i}(k)}{\partial \underline{x}_{i}(k)}\right)^{T} \underline{\beta}_{j}(k)+\left(\frac{\partial \underline{f}_{i}(k)}{\partial \underline{z}_{i}(k)}\right)^{T} \underline{\lambda}_{i}(k) \\
k=n, n & -1, \cdots, 0
\end{aligned}
$$

4. Calculate, $\frac{\partial L_{i}}{\partial \underline{u}_{i}(k)}$ and $\frac{\partial L_{i}}{\partial \underline{z}_{i}(k)}$ using $\underline{x}_{i}(k)$ and $\underline{\lambda}_{i}(k)$.

5. Update, $\underline{u}_{i}(k)$ and $\underline{z}_{i}(k)$ by relations given bellow:

$\underline{u}_{i}^{(l+1)}(k)=\underline{u}_{i}^{(l)}(k)-\rho \frac{\partial L_{i}}{\partial \underline{u}_{i}(k)}$

$$
\underline{z}_{i}^{(l+1)}(k)=\underline{z}_{i}^{(l)}(k)-\rho \frac{\partial L_{i}}{\partial \underline{z}_{i}(k)}
$$

6. If $\sum_{k=0}^{n+1}\left\|\frac{\partial L_{i}}{\partial \underline{z}_{i}(k)}\right\|^{2}+\sum_{k=0}^{n}\left\|\frac{\partial L_{i}}{\partial \underline{u}_{i}(k)}\right\|^{2}<\varepsilon$ stop the algorithm, else go to step (2).

\section{COORDINATION USING THE GRADIENT OF ERRORS}

In the second level the goal is to update $\beta$, in order to decrease the interaction errors defined as

$$
\underline{e}_{i}(k)=\underline{z}_{i}(k)-H_{i}(\underline{x}(k))
$$

In old methods of coordination, gradient of overall performance function was used for coordination [3],[4]. In this paper, gradient of interaction error is used for coordination. This method first proposed by Sadati [6]-[8], has faster convergence rate than previous ones.

Because the gradient of interaction error is used for coordination, we should find the gradient of interaction error related to the coordination parameters.

Let $\underline{x}_{i}(k), \underline{u}_{i}(k), \underline{z}_{i}(k)$ and $\underline{\lambda}_{i}(k)$ be the optimum values provided by the first level with previous $\beta$. These values satisfy the necessary optimality conditions at the first level. i.e.

$$
\frac{\partial L_{i}}{\partial \underline{x}_{i}}=\underline{0} ; \quad \frac{\partial L_{i}}{\partial \underline{u}_{i}}=\underline{0} ; \quad \frac{\partial L_{i}}{\partial \underline{z}_{i}}=\underline{0} ; \quad \frac{\partial L_{i}}{\partial \underline{\lambda}_{i}}=\underline{0}
$$

Now the necessary conditions in (16), can be shown by the following equation

$$
\frac{\partial L_{i}\left(\underline{w}_{i}, \underline{\beta}\right)}{\partial \underline{w}_{i}}=\underline{0}
$$

where

$$
\underline{w}_{i}=\left[\begin{array}{c}
\underline{X}_{i} \\
\underline{U}_{i} \\
\underline{Z}_{i} \\
\underline{\Lambda}_{i}
\end{array}\right] \quad \underline{\beta}=\left[\begin{array}{c}
\underline{\mathrm{B}}_{1} \\
\vdots \\
\underline{\mathrm{B}}_{N}
\end{array}\right] \quad ; i=1,2, \cdots, N
$$

and

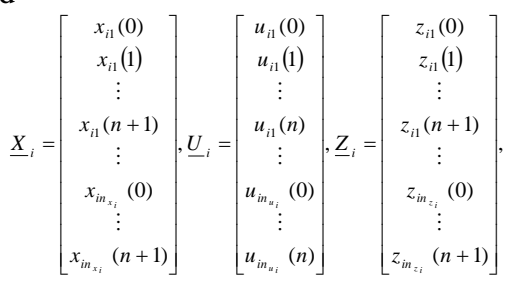

$$
\underline{\Lambda}_{i}=\left[\begin{array}{c}
\lambda_{i 1}(0) \\
\lambda_{i 1}(1) \\
\vdots \\
\lambda_{i 1}(n) \\
\vdots \\
\lambda_{i n_{x i}}(0) \\
\vdots \\
\lambda_{i n_{x i}}(n)
\end{array}\right], \underline{B}_{i}=\left[\begin{array}{c}
\beta_{i 1}(0) \\
\beta_{i 1}(1) \\
\vdots \\
\beta_{i 1}(n+1) \\
\vdots \\
\beta_{i n_{21}}(0) \\
\vdots \\
\beta_{i n_{2 i}}(n+1)
\end{array}\right]
$$

Since each $L_{i}$ is independent of $\underline{W}_{j}$ s, for $i \neq j$, we get

$$
\frac{\partial L_{i}\left(\underline{w}_{i}, \underline{\beta}\right)}{\partial \underline{w}_{j}}=\underline{0} \quad i \neq j
$$

so

$$
\frac{\partial L(\underline{w}, \underline{\beta})}{\partial \underline{w}}=L_{\underline{w}}(\underline{w}, \underline{\beta})=\underline{0}
$$

where

$$
\underline{w}=\left[\begin{array}{c}
\underline{w}_{i} \\
\underline{w}_{2} \\
\vdots \\
\underline{w}_{N}
\end{array}\right]
$$

and

$$
L(\underline{w}, \underline{\beta})=\sum_{i=1}^{N} L_{i}\left(\underline{w}_{i}, \underline{\beta}\right)
$$

Now, by consideration of small variations in $\underline{w}$ and $\beta$, the following equations result from (19) 


$$
\frac{\partial L_{\underline{w}}(\underline{w}, \underline{\beta})}{\partial \underline{w}} \delta \underline{w}+\frac{\partial L_{\underline{w}}(\underline{w}, \underline{\beta})}{\partial \underline{\beta}} \delta \underline{\beta}=\underline{0}
$$

or

$$
L_{\underline{w} \underline{w}} \delta \underline{w}+L_{\underline{w} \underline{\beta}} \delta \underline{\beta}=\underline{0}
$$

Now if $\delta \underline{\beta}$ and $\delta \underline{w}$ become very close to zero, $\frac{\partial \underline{w}}{\partial \underline{\beta}}$ can be written as bellow

$$
\frac{\partial \underline{w}}{\partial \underline{\beta}}=-L_{\underline{w} \underline{w}}^{-1}(\underline{w}, \underline{\beta}) L_{\underline{w} \underline{\beta}}(\underline{w}, \underline{\beta})
$$

Our goal is to calculate $\frac{\partial \underline{e}}{\partial \underline{\beta}}$, where $\underline{e}$ is the interaction error resulted by

$$
\underline{e}=\left[\begin{array}{c}
\underline{e}_{1} \\
\underline{e}_{2} \\
\vdots \\
\underline{e}_{N}
\end{array}\right] ; \underline{e}=\underline{z}-\underline{z}^{*}
$$

The interaction error vector can be defined as

$$
\underline{e}_{i}(k)=\underline{z}_{i}(k)-H_{i}(\underline{x}(k))
$$

where

$$
\underline{Z}=\left[\begin{array}{c}
\underline{Z}_{1} \\
\vdots \\
\underline{Z}_{N}
\end{array}\right] \quad, \quad \underline{Z}^{*}=\left[\begin{array}{c}
\underline{Z}_{1}^{*} \\
\vdots \\
\underline{Z}_{N}^{*}
\end{array}\right]
$$

and

$$
\underline{Z}_{i}^{*}(k)=H_{i}\left(\underline{x}_{1}(k), \cdots, \underline{x}_{N}(k)\right)=\sum_{i=1}^{N} H_{i j}\left(\underline{x}_{j}(k)\right)
$$

Now, $\frac{\partial \underline{e}}{\partial \underline{\beta}}$ can be written as follows

$$
\frac{\partial \underline{e}}{\partial \underline{\beta}}=\frac{\partial \underline{z}}{\partial \underline{\beta}}-\frac{\partial \underline{z}^{*}}{\partial \underline{x}} \cdot \frac{\partial \underline{x}}{\partial \underline{\beta}}
$$

In these equations, $\frac{\partial \underline{z}}{\partial \underline{\beta}}$ and $\frac{\partial \underline{x}}{\partial \underline{\beta}}$ can be easily calculated from $\frac{\partial \underline{w}}{\partial \beta}$. For a detailed calculation of $L_{\underline{w} \underline{\beta}}$ and $L_{\underline{w} \underline{w}}$, one can refer to [8].

The sum-squared error is also given by

$$
S E=\frac{1}{2} \underline{e}^{T} \underline{e}
$$

So, the coordination parameter, $\beta$, can be updated by the following equation

$$
\underline{\beta}^{(l+1)}=\underline{\beta}^{(l)}-\eta\left(\frac{\partial \underline{e}}{\partial \underline{\beta}}\right)^{T} \underline{e}
$$

In this equation, $\eta$ is the step length and $l$ is the iteration index.

Now by using the following algorithm, optimization of large-scale dynamic systems using Interaction Balance Principle and gradient-based coordinator is possible.

1. Start with initial values for coordination parameters.

2. Solve the first level optimization problems with these known coordination parameters using gradient method and calculate $\underline{x}_{i}, \underline{u}_{i}, \underline{Z}_{i}$ and $\underline{\lambda}_{i}$.

3. Calculate the interaction errors and the gradient matrix by (25a) and (23).

4. Update $\beta$ by (28).

5. Calculate the sum-squared error. If it is smaller than a desired value terminate the algorithm, else go to step (2).

This two-level algorithm can be shown as block diagram in Fig. 2.

\section{SIMULATION RESULTS}

As a system for simulation, similar to Part I, a system composed of 4 connected water tanks is chosen [9]. This system is shown in Fig. 3.

Choosing $\left[\begin{array}{ll}h_{1} & h_{2}\end{array}\right]^{T}$ as $\underline{h}_{1}$ and $\left[\begin{array}{ll}h_{3} & h_{4}\end{array}\right]^{T}$ as $\underline{h}_{2}$, the discrete time state space equations for this system can be given as

$$
\begin{aligned}
& \underline{h}_{1}(k+1)=\underline{h}_{1}(k)+A_{1} \sqrt{\underline{h}_{1}(k)}+B_{1} u_{1}(k)+C_{1} \sqrt{z_{1}(k)} \\
& \underline{h}_{2}(k+1)=\underline{h}_{2}(k)+A_{2} \sqrt{\underline{h}_{2}(k)}+B_{2} u_{2}(k)+C_{2} \sqrt{z_{2}(k)}
\end{aligned}
$$

The unknown parameters of the above equations are listed bellow :

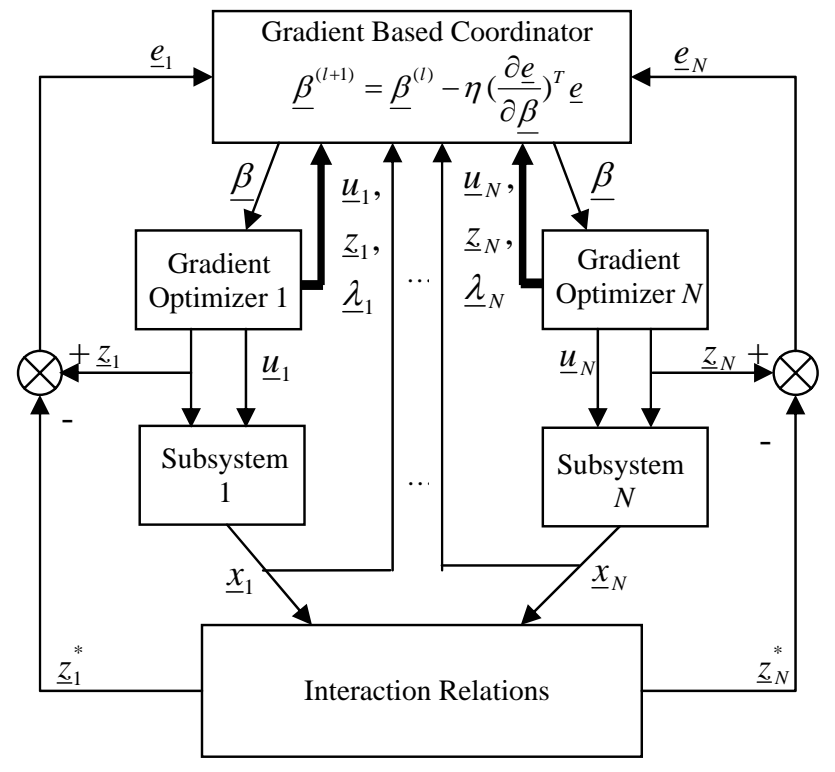

Fig. 2 Two-level Coordination based on gradient of interaction error 


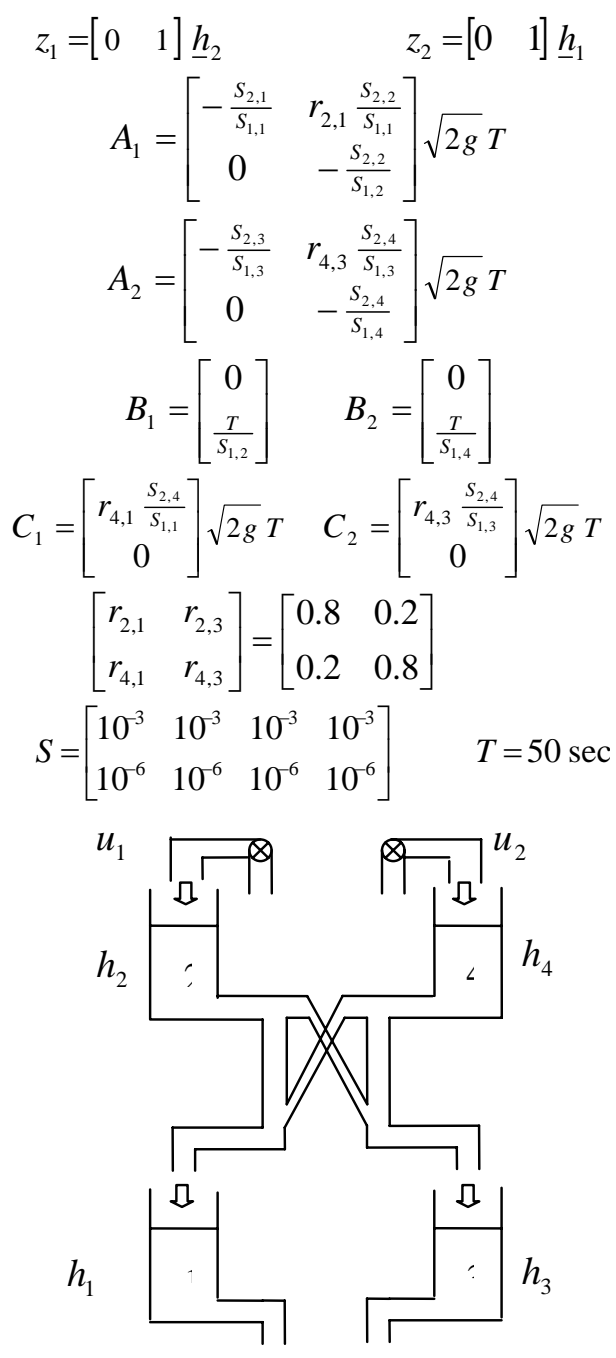

Fig. 3 Connected 4 water tanks

Now, the goal is to move the water height of all the tanks to $1 \mathrm{~m}$., so that a cost function like (30) is minimized.

$$
\begin{aligned}
J= & \frac{1}{2}\left\|\underline{h}_{1}(N)-\underline{h}_{1 f}\right\|_{Q_{1}}^{2}+\frac{1}{2}\left\|\underline{h}_{2}(N)-\underline{h}_{2 f}\right\|_{Q_{2}}^{2} \\
& +\sum_{k=0}^{N-1}\left[\frac{1}{2}\left\|\underline{h}_{1}(k)-\underline{h}_{1 f}\right\|_{Q_{1}}^{2}+\frac{1}{2}\left\|u_{1}(k)-u_{1 f}\right\|_{R_{1}}^{2}\right] \\
& +\sum_{k=0}^{N-1}\left[\frac{1}{2}\left\|\underline{h}_{2}(k)-\underline{h}_{2 f}\right\|_{Q_{2}}^{2}+\frac{1}{2}\left\|u_{2}(k)-u_{2 f}\right\|_{R_{2}}^{2}\right] \\
& Q_{1}=Q_{2}=\left[\begin{array}{ll}
1 & 0 \\
0 & 1
\end{array}\right] \quad, R_{1}=R_{2}=10^{11} \\
& \underline{h}_{1 f}=\underline{h}_{2 f}=\left[\begin{array}{l}
1 \\
1
\end{array}\right] \quad, u_{1 f}=u_{2 f}=4.4294 \times 10^{-6}
\end{aligned}
$$

This problem is solved by the proposed two-level method using both the old and the new methods of coordination.

For comparison of the two coordination methods (old method and the new coordination method based on the gradient of errors), sum-squared of interaction errors is computed and shown in Fig. 4. As it can be seen, the new approach has faster convergence rate than the classical method.

Optimal inputs and states, computed by this new twolevel method, are shown in Figs. 5 and 6, which also show the stability of the system.

For showing the capability of the new two-level method, the problem is also solved by a centralized approach. The differences between the solutions are depicted in Fig. 7. The small values of errors show the capability and optimality of the new two-level method.

\section{CONCLUSION}

In this paper, a new two-level method has been used for optimal control of large-scale systems. In this approach, the first level optimization problems are solved using an iterative gradient based method and in the second level, a coordinator uses the gradient of coordination errors to update the coordination parameters. As it is seen, the new approach has faster convergence rate than the classical Goal Coordination approach.

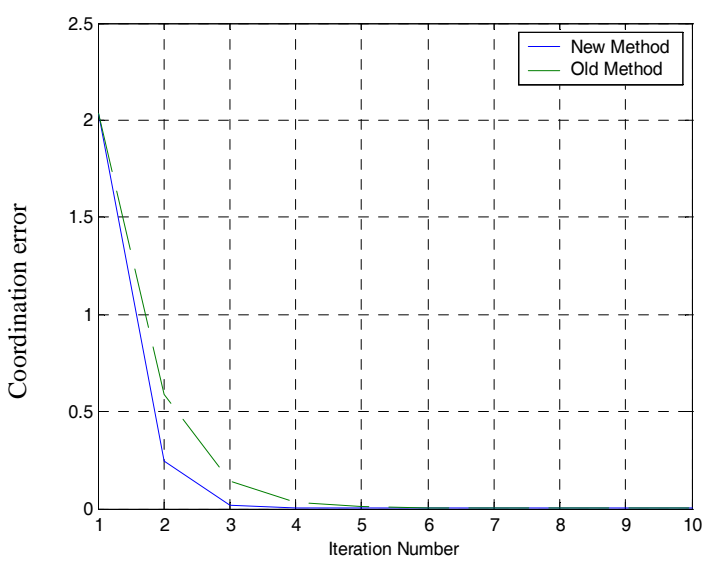

Fig. 2 Coordination errors for both coordination methods (solid: New gradient based approach, dashed: Classical coordination method)
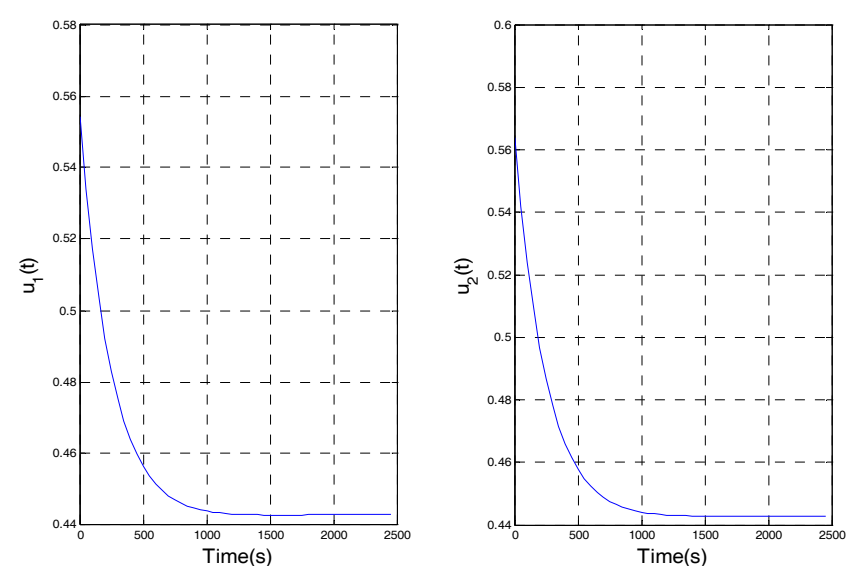

Fig. 5 Optimal inputs computed by the new two-level method 

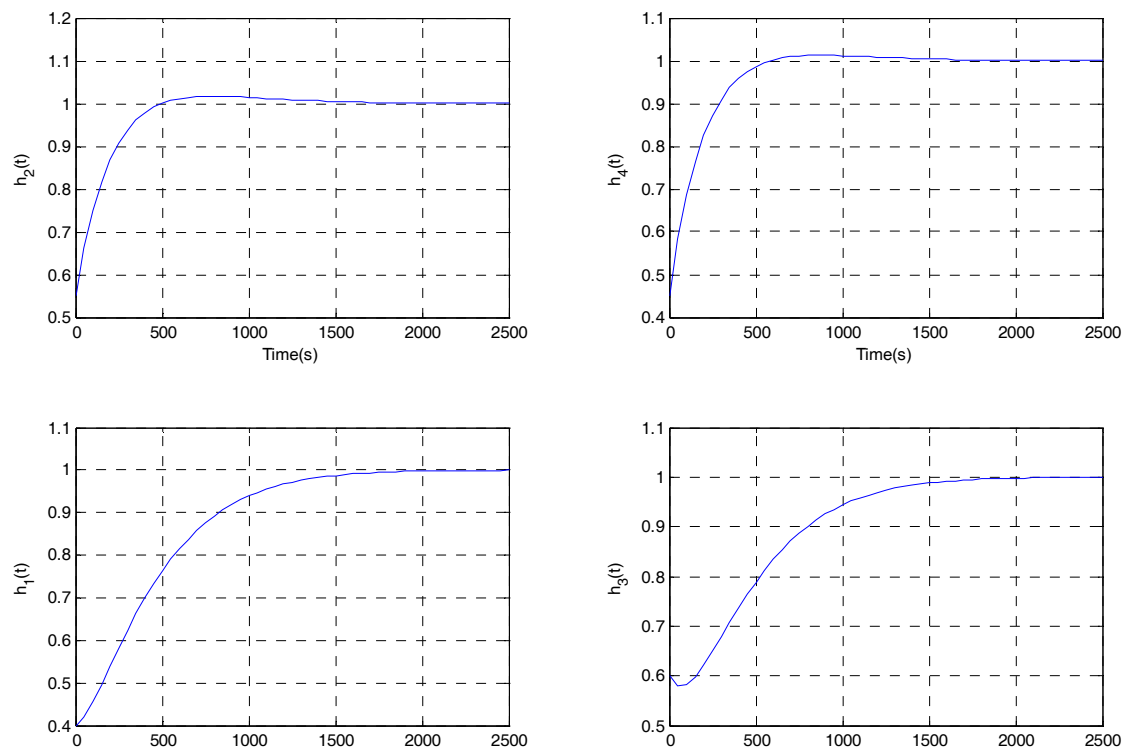

Fig. 6 Optimal states responses using the new goal coordination approach
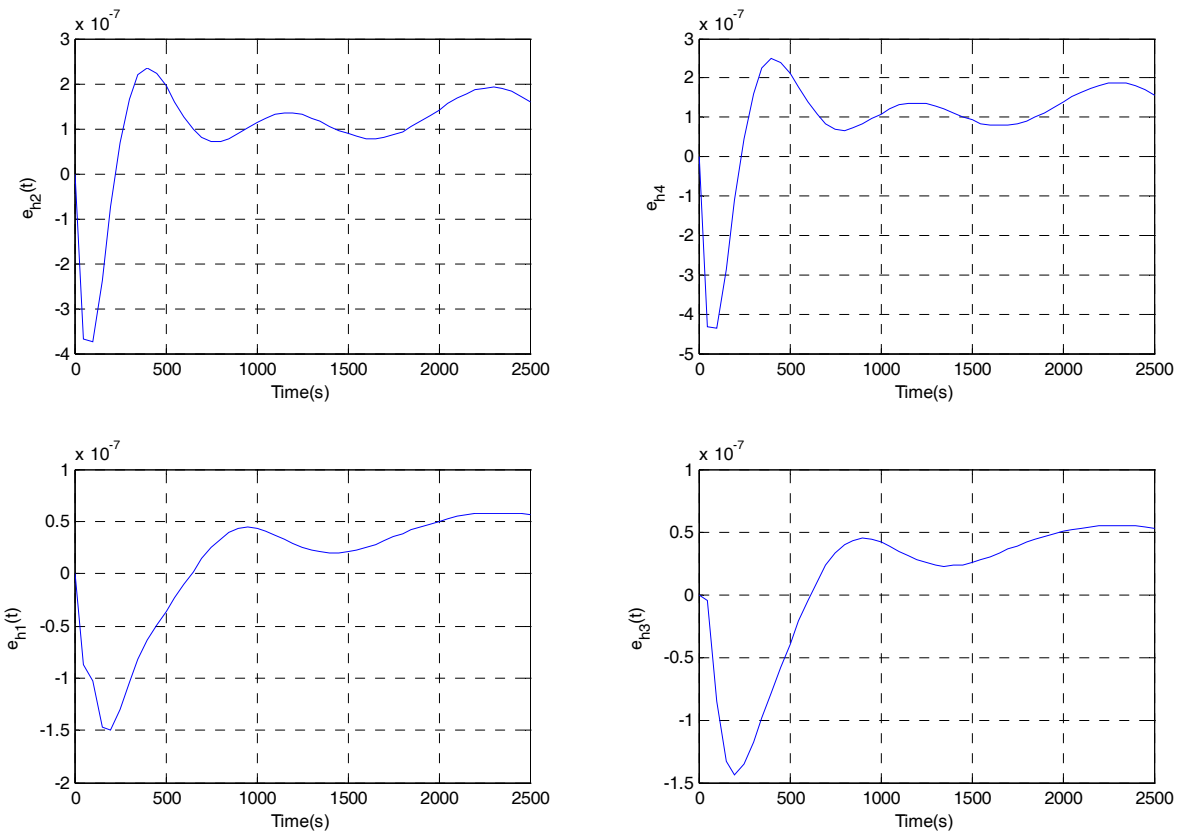

Fig. 7 comparison of errors using the new coordination approach and the centralized solution

\section{REFERENCES}

[1] M. D. Mesarovic, D. Macko and Y. Takahara, "Two coordination principles and their applications in large-scale systems control," in Proc. IFAC Congr., Warsaw, Poland, 1969.

[2] - Theory of Hierarchical Multilevel Systems. New York: Academic Press, 1970

[3] M. D. Singh, Dynamical Hierarchical Control. Rev. Ed., Amsterdam, North Holland, 1980.

[4] M. Jamshidi, Large-Scale Systems: Modeling, Control and Fuzzy Logic. Prentice Hall, 1997.

[5] J. D. Pearson, Dynamic Decomposition Techniques in Optimization Methods for Large Scale Systems. D. A. Wismer (Editor), McGraw Hill, 1971.
[6] N. Sadati, "A gradient based method for goal coordination of largescale systems," Sharif University of Technology, Electrical Engineering Department, Report no. SUT-ICSL(19), 1997.

[7] N. Sadati, "A new two-level gradient based approach for intelligent coordination of large-scale systems; Part II - Interaction balance principle,” Sharif University of Technology, Electrical Engineering Department, Report no. SUT-ICSL(31), 2000.

[8] N. Sadati, "A novel approach to coordination of large-scale systems; Part II-Interaction balance principle,” in press, IEEE International Conference on Industrial Technology, Hong Kong, Dec. 2005.

[9] J.A. Roubos, S. Mollov, R. Babuska and H.B. Vertruggen, "Fuzzy model-based Predictive control using Takagi-Sugeno models," Int. J. Approx. Reason, vol. 22, 1999, pp. 3-30. 